\title{
A utilização da fitoterapia e plantas medicinais como prática terapêutica pela visão de estudantes de enfermagem
}

\author{
The use of phytotherapy and medicinal plants as therapeutic practice by \\ the view of nursing students.
}

Daniela Pereira Alves ${ }^{1}$, Eva Jessica de Jesus Silva ${ }^{1}$, Maronne Quadros Antunes ${ }^{1}$, Eurislene Antunes Damasceno ${ }^{1}$, Patrícia de Oliveira Lima ${ }^{2}$, Ricardo Lopes Rocha ${ }^{2}$, Marcos Luciano Pimenta Pinheiro².

${ }^{1}$ Faculdades Unidas do Norte de Minas - FUNORTE, Montes Claros, Minas Gerais, Brasil.

${ }^{2}$ Universidade Federal dos Vales do Jequitinhonha e Mucuri - UFVJM, Diamantina, Minas Gerais, Brasil.

\section{Resumo}

Introdução: O saber popular e o conhecimento empírico há muito vêm demonstrando os efeitos benéficos proporcionados pelo uso de plantas como terapia complementar ou alternativa. Objetivo: Este estudo teve como objetivo avaliar o conhecimento e o uso de plantas medicinais e fitoterápicos como prática terapêutica por acadêmicos de enfermagem. Metodologia: Trata-se de um estudo transversal, de caráter quantitativo, composto por uma mostra de 90 acadêmicos de enfermagem, utilizando um questionário semiestruturado adaptado para a coleta de dados. Resultados: Nenhum entrevistado conhecia a diferença de conceito entre fitoterápico e planta medicinal e $67 \%$ destes, afirmaram saber pouco sobre o assunto, mas a totalidade dos entrevistados afirmou que já utilizaram plantas medicinais pelo menos uma vez, principalmente na forma de chá. Conclusão: Conclui-se que há uma lacuna no aprendizado dos acadêmicos de enfermagem sobre o conhecimento da fitoterapia e das plantas medicinais. A inserção de uma disciplina com esta temática poderá contribuir para a solução deste problema, melhorando o conhecimento na área.

Palavras-Chave: Medicamentos Fitoterápicos; Conhecimento; Educação em Enfermagem.

Autor correspondente:

Marcos Luciano Pimenta Ribeiro

Endereço: Rua da Glória, 187, Centro Lab. de Farmacologia Campus I

CEP 39100-000. Diamantina (MG), Brasil.

Telefone: (38) 988051361

Recebido em: 25/06/2019

Revisado em: 09/08/2018

Aceito em: 28/06/2019

E-mail: marcospimenta2@gmail.com

Publicado em: 30/09/2019 


\begin{abstract}
Introduction: Popular knowledge and empirical knowledge have long been demonstrating the beneficial effects afforded by the use of plants as complementary or alternative therapy. Objective: The objective of this study was to evaluate the knowledge and the use of medicinal plants and phytotherapics as therapeutic practice by nursing students. Methods: It is a cross-sectional, quantitative study, composed of a sample of 90 nursing students, using a semi-structured questionnaire adapted for the data collect. Results: No interviewee knew the difference in concept between herbal and medicinal plants and 67\% said they knew little about the subject, but the totality of the interviewees stated that they have used medicinal plants at least once, mainly in the form of tea. Conclusion: It is concluded that there is a gap in the nursing students' knowledge about the knowledge of phytotherapics and medicinal plants. The insertion of a discipline with this theme may contribute to the solution of this problem, improving knowledge in the area.
\end{abstract}

Keywords: Phithoterapeutic Drugs; Knowledge; Education, Nursing

\title{
Introdução
}

A fitoterapia é utilizada desde tempos remotos, quando o homem experimentava espécies vegetais para tratar suas enfermidades de maneira empírica por meio da observação dos seus efeitos ${ }^{1}$. Existem relatos acerca do Suo Uen, um tratado escrito há mais de 5 mil anos que catalogou aproximadamente 268 ervas e serve como base para a fitoterapia oriental ${ }^{2}$.

O saber popular e o conhecimento empírico há muito vêm demonstrando os efeitos benéficos proporcionados pelo uso de plantas como terapia complementar ou alternativa. Esta realidade aproxima-se cada vez mais da literatura científica, possibilitando a comprovação dos efeitos dessa prática. Desta maneira é importante que os profissionais da saúde se atualizem nesta área ${ }^{3}$. Além disso, a fitoterapia pode e deve ser considerada como campo de interação, pois valoriza a prática e saberes locais, por meio do envolvimento dos usuários com os recursos naturais e pela interação dos profissionais da equipe de saúde ${ }^{4}$.

Nesse contexto, a partir do ano de 2006, foram instituídas no Brasil ações nas políticas de saúde que reconheceram e promoveram a introdução do uso de plantas medicinais e dos fitoterápicos no Sistema Único de Saúde ${ }^{5,6}$. Já existe a prática da fitoterapia em várias regiões do Brasil, em fase de implantação ou implantada ${ }^{4,7}$, o que facilita o acesso da população às plantas medicinais, enriquecendo o conhecimento de todos os envolvidos ${ }^{7}$.

Entretanto, é necessário mais orientação na área, para que tal estratégia possa beneficiar a população assistida de forma correta e segura ${ }^{8}$, com práticas de educação continuada ${ }^{9}$. Outro obstáculo encontrado para a efetivação dessa prática na Atenção Básica à Saúde encontra-se nas estruturas físicas e nas escassas capacitações profissionais ${ }^{10}$, além disso, as pesquisas publicadas com a utilização desse recurso terapêutico para o tratamento de doenças ainda são reduzidas ${ }^{11,12}$, apesar da grande biodiversidade ${ }^{13}$ e da riqueza cultural sobre plantas medicinais ${ }^{14}$ existente existentes no Brasil.

Qualificar os profissionais atuantes na atenção primária e ampliar o acesso à fitoterapia como prática alternativa e complementar podem ser uma opção para melhorar essa estratégia de tratamento ${ }^{10}$.

Assim, esta pesquisa teve como objetivo analisar o interesse e a percepção de acadêmicos de enfermagem sobre a prática fitoterápica como forma 
terapêutica, a fim de planejar futuras ações de educação continuada acerca do tema.

\section{Metodologia}

Trata-se de um estudo transversal com abordagem quantitativa realizado durante o mês de setembro de 2016, aprovado pelo Comitê de Ética e Pesquisa das Faculdades Integradas do Norte de Minas sob o parecer $\mathrm{n}^{\circ} 1.742 .823 / 2016$, de acordo com os princípios estabelecidos pela Resolução $n^{\circ} n^{\circ}$ 466/20 2012 do Conselho Nacional de Saúde ${ }^{15}$.

A amostra de conveniência foi composta por acadêmicos matriculados no segundo semestre de 2016 no curso de graduação em Enfermagem de uma instituição privada na cidade de Montes Claros, região norte de Minas Gerais.

Os critérios de inclusão foram: o participante estar matriculado no primeiro, quinto ou décimo período do curso, com idade igual ou superior a 18 anos, além de aceitar participar de forma voluntária da pesquisa, assinando o Termo de Consentimento Livre e Esclarecido (TCLE). Os participantes ausentes no dia da aplicação do questionário foram excluídos da pesquisa.

Para a coleta dos dados foi utilizado um questionário adaptado ${ }^{16}$. Realizou-se estatística descritiva simples por meio do programa Statistical Package for the Social Sciences (SPSS) versão 20.0.

\section{Resultados e Discussão}

A amostra contemplou um total de 90 acadêmicos de ambos os gêneros com faixa etária entre 18 e 80 anos, na qual foi observada uma média de idade de 28 anos, sendo a maioria do sexo feminino $(75,4 \%)$. Essa predominância também foi relatada nos estudos de Bruning et al. (2012) e Feitosa et al. (2016) $)^{5,17}$, que reportaram taxas de $80 \%$ e $69,8 \%$, respectivamente.

Em relação à diferença entre o conceito de fitoterápicos e plantas medicinais, todos os entrevistados disseram não saber, possivelmente por não haver disciplina sobre este assunto no referido curso. Estudos semelhantes realizados com 74 estudantes do curso de nutrição, numa universidade privada de Teresina $-\mathrm{PI}^{18}$, e com 94 estudantes do curso de enfermagem de uma Instituição de Ensino Superior de Brasília - $\mathrm{DF}^{19}$, demonstraram que $75,68 \%$ e $41,5 \%$ dos acadêmicos, respectivamente, afirmaram conhecer o conceito de fitoterapia, fato que também pode ser devido à ausência de disciplina acerca do assunto. Fato também observado Em uma pesquisa realizada com 96 profissionais da Estratégia de Saúde da Família (Cirurgiões-Dentistas, Enfermeiros, Farmacêuticos, Médicos e Nutricionistas) no município de Petrolina - PE, 67\% dos entrevistados também relataram não saber a diferença entre fitoterápicos e homeopáticos ${ }^{20}$, e no estudo de Ferreira et al. (2019), observou-se que há enfermeiros que utilizam plantas medicinais e fitoterápicos com pouco conhecimento das práticas integrativas e complementares, impedindo-os de esclarecer dúvidas dos pacientes ${ }^{21}$, o que é preocupante, posto que são profissionais que são acionados pelos usuários do serviço de saúde para obtenção de informações sobre o assunto. Em relação ao conhecimento sobre a fitoterapia, $67 \% \quad(n=60)$ dos acadêmicos afirmaram saber pouco sobre o assunto. Resultado próximo a esse foi encontrado no estudo Faria et al. $(2017)^{22}$, que avaliaram o conhecimento sobre fitoterapia entre os acadêmicos da faculdade de Ciências da Vida de uma universidade da Região Norte do Brasil, onde 58\% dos entrevistados concordaram que não possuem conhecimento suficiente a respeito deste tema.

Quando questionados quanto à utilização de plantas medicinais, todos os estudantes afirmaram que já utilizaram pelo menos uma vez; sendo que, as mais citadas foram Camomila (24\%), Capim Santo (18\%), Alho (17\%) e Boldo do Chile (14\%), provavelmente devido ao fácil acesso de tais plantas pelos usuários, e também por possuírem ações farmacológicas como auxiliar nos problemas digestivos, calmantes e anti-inflamatórios, que são problemas frequentemente enfrentados pelos estudantes e corrobora os dados das pesquisas de Moura et al. (2016), Zeni et al. (2017), Ângelo e Ribeiro (2014) e Costa et al. (2010) $)^{18,23-25}$ que citaram, respectivamente 
A utilização da fitoterapia e plantas medicinais como prática terapêutica pela visão de estudantes de enfermagem

em seus estudos, a Camomila, o Capim Santo, o Alho e o Boldo do Chile, como as plantas mais utilizadas pelos seus entrevistados,

As formas mais frequentemente relatadas para a utilização de plantas medicinais no nosso estudo foram: chá; principalmente por infusão $72 \%(\mathrm{n}=65)$, xarope $11 \%(n=10)$, gargarejos $11 \%(n=10)$, e garrafadas 6\% $(\mathrm{n}=5)$ (FIGURA 1), possivelmente por ser o método mais fácil e econômico de utilização de tais plantas. Resultado similar, indicando o chá como a forma mais usada, também foi reportado por Zeni et al. (2017) e por Lopes e Pantoja (2012) 23,26 . De acordo com estudos prévios, o chá por infusão é o principal meio de preparo das plantas medicinais ${ }^{10}$.

\section{$\square$ Chá de Infusão $\square$ Garrafadas $\square$ Gargarejo $\square$ Xarope}

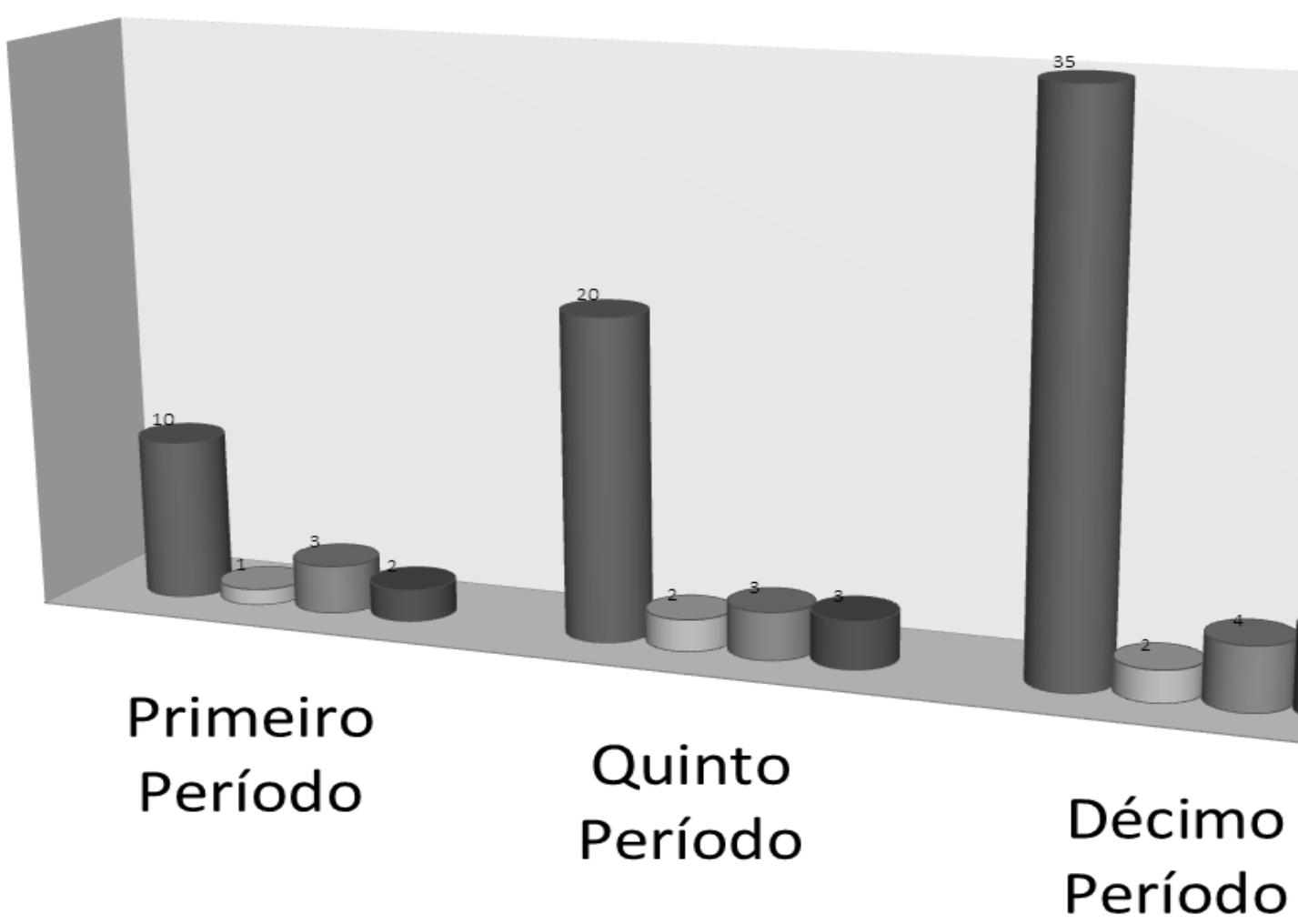

FIGURA 1: Frequência (n) relativa às formas de uso de fitoterápicos e/ou plantas medicinais de acordo com o período do curso.

Quanto ao uso dos medicamentos fitoterápicos, a matéria prima vegetal em forma de pó foi a mais utilizada (FIGURA 2), discordando dos resultados do estudo de Araújo et al., (2015), realizado em seis
Unidades Básicas de Saúde do município de Parnamirim, RN, no qual relatou-se que a população estudada utiliza as folhas para fazer chás ${ }^{27}$. 


\section{Pó $\square$ Pomada $\square$ Comprimido Cápsula}

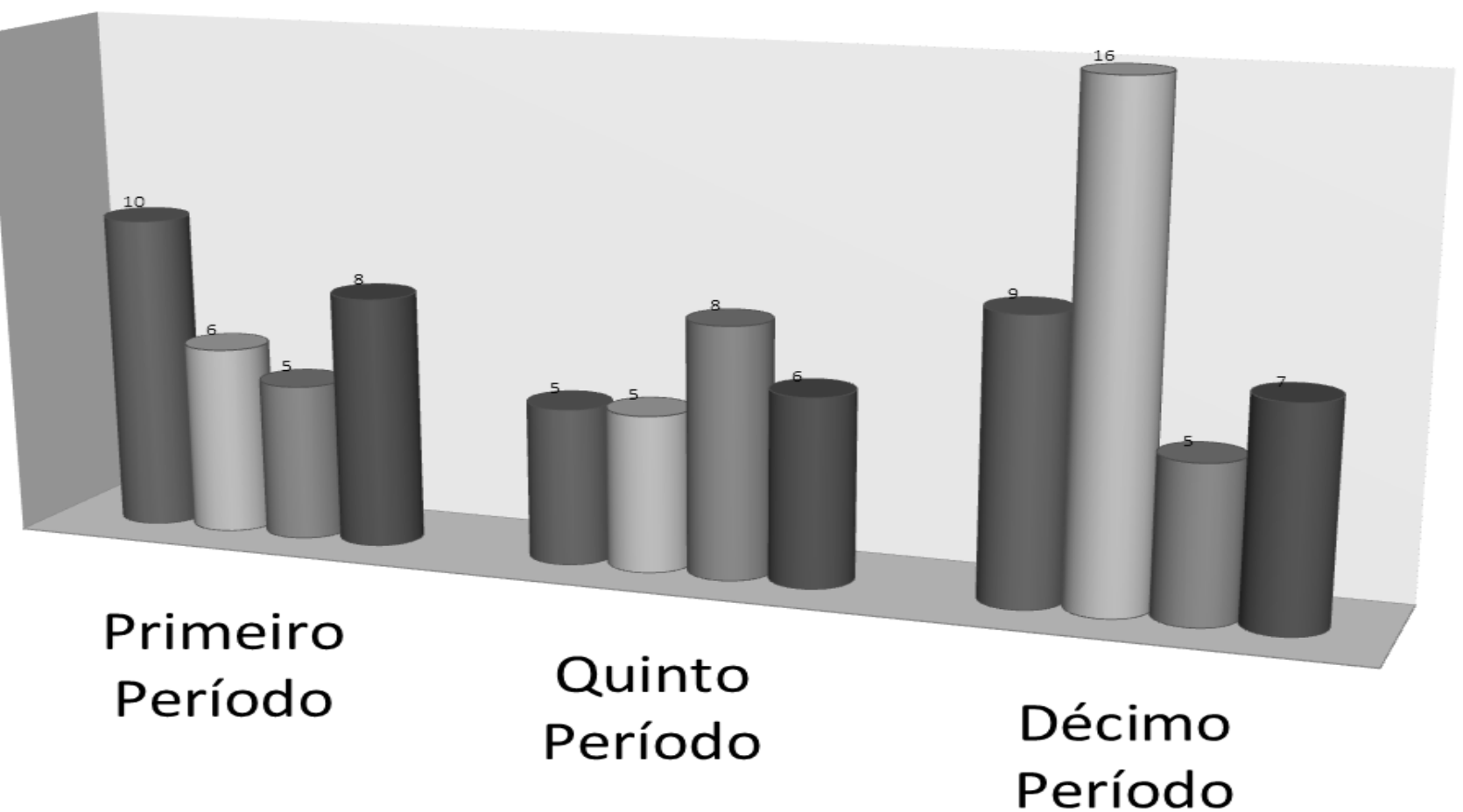

FIGURA 2: Frequência (n) relativa ao uso de formas farmacêuticas de medicamentos fitoterápicos, de acordo com o período do curso.

Em relação à frequência de uso das plantas medicinais e/ou fitoterápicos, os resultados mostraram que $22 \%$ dos entrevistados $(n=20)$ fazem uso anual, $50 \%$ $(n=45)$ uso mensal, $17 \% \quad(n=15)$ uso semanal e $11 \%$ $(\mathrm{n}=10)$ uso diário (FIGURA 3). Valores superiores aos nossos, foram relatados num estudo realizado no município de Itaipulândia - PR, com pacientes idosos acompanhados pela atenção primária onde foi observado o uso semanal e diário de plantas medicinais por $22,7 \%$ e $30,5 \%$ dos entrevistados respectivamente ${ }^{28}$. 


\section{- Primeiro Período $\square$ Quinto Período $\square$ Décimo Período}

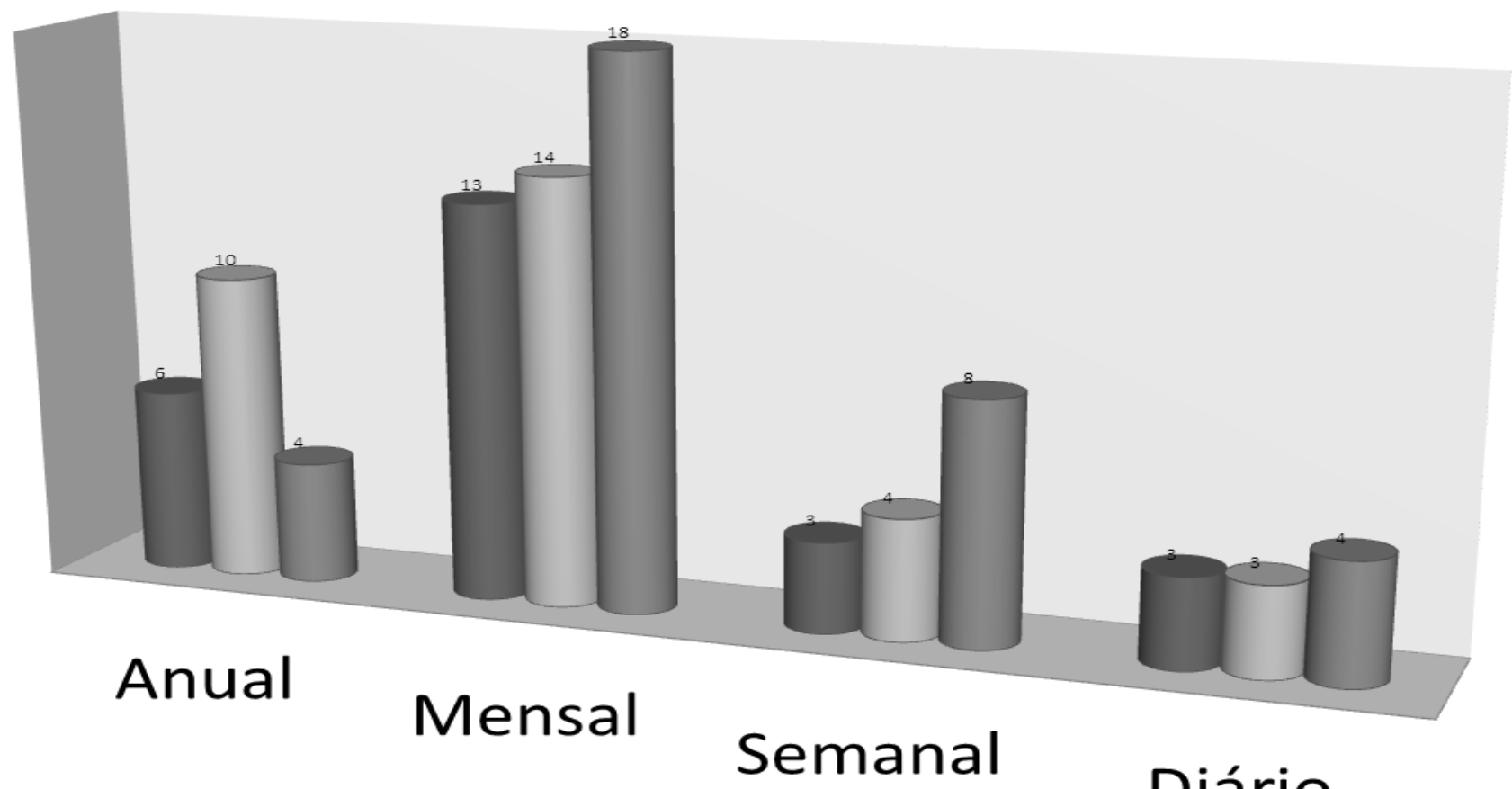

FIGURA 3: Frequência (n) de uso de fitoterápicos e/ou plantas medicinais, de acordo com o período do curso.

De acordo com os dados coletados, os medicamentos fitoterápicos e as plantas medicinais são provenientes do cultivo em casa $44 \%(n=40)$, de raizeiros $22 \%(n=20)$, das hortas $17 \%(n=15)$, da pastoral da saúde $6 \%(n=5)$, dos quintais dos vizinhos $6 \% \quad(n=5)$, de ambulantes 3\% $(\mathrm{n}=3)$ ou adquiridos com médicos $2 \%$ $(n=2)$. Resultados semelhantes foram relatados por Ceolin et al. $(2013)^{10}$ que afirmaram que os entrevistados de seu estudo obtiveram as plantas na própria casa (27\%) e em ervanários (19\%).

A maioria dos entrevistados aprendeu a utilizar as plantas medicinais pesquisando a internet $33 \%$ $(n=30)$, aprendendo com os avós $27 \%(n=25)$, dentre outras maneiras. Um relato de experiência sobre o uso de plantas medicinais em uma comunidade do município de Uruguaiana - RS mostrou resultados diferentes onde a maioria dos entrevistados $(75,5 \%)$ aprendeu sobre as plantas medicinais com a família e $15,6 \%$ com a pastoral da saúde ${ }^{29}$.

Quando questionados sobre o conhecimento da Política Nacional de Plantas Medicinais e Fitoterápicos, todos os entrevistados afirmaram não conhecer o assunto. Resultados próximos aos nossos foram encontrados num estudo realizado com 240 acadêmicos dos cursos da área de saúde de uma universidade de Montes Claros - MG., no qual $81,3 \%$ dos entrevistados relataram não conhecer o assunto $^{17}$. Com isso, pode-se deduzir que este assunto é pouco abordado durante a graduação, o que pode tornar deficitária a formação dos futuros profissionais em relação a esta temática. Em uma revisão de literatura publicada recentemente, os autores avaliaram a importância da informação do profissional de enfermagem sobre o cuidado no uso das plantas medicinais ${ }^{30}$. Pesquisas realizadas na Austrália ${ }^{31}$ e nos Estados Unidos $^{32}$, relataram estratégias para a inserção do conhecimento e uso das práticas integrativas e complementares na formação dos enfermeiros. Em uma revisão sistemática publicada por Balouchi e colaboradores (2018) afirmam que até o presente momento, não se pode precisar o quanto esse conhecimento faz parte da grade curricular dos cursos de enfermagem. Revela ainda que nos estudos avaliados, as práticas integrativas e complementares mais utilizadas 
por estes profissionais foram a fitoterapia, terapias mentecorpo e aromaterapia ${ }^{33}$.

Alguns gestores apontam como dificuldade encontrada no serviço de saúde a aceitação desta prática terapêutica pelos profissionais de saúde ${ }^{34}$. No presente estudo, observou-se o oposto, pois foi constatado grande interesse por parte dos entrevistados, futuros profissionais da saúde. Do total de acadêmicos participantes da coleta, 98\% $(n=88)$ disseram ter interesse em estudar esse tema, caso fosse ofertada uma disciplina na grade curricular. Resultados semelhantes foram relatados por Feitosa et al. $(2016)^{17}$, em uma pesquisa com estudantes da área de saúde, onde $70,8 \%$ dos entrevistados demonstraram interesse em incluir a disciplina de fitoterapia em sua graduação. Os profissionais de enfermagem devem ser estimulados a ter interesse pelas práticas terapêuticas na graduação, redefinindo assim os conceitos propostos de assistência à saúde mais integralizada ${ }^{8,35}$.

\section{Conclusão}

A pesquisa aponta uma lacuna no aprendizado dos acadêmicos de enfermagem sobre o conhecimento da fitoterapia e das plantas medicinais, visto que no curso avaliado não há uma disciplina que contemple tal temática. A inserção desta poderá contribuir para a solução desse problema, melhorando o conhecimento na área.

\section{Declaração de Conflitos de Interesse}

Os autores do artigo afirmam que não houve nenhuma situação de conflito de interesse, tais como propostas de financiamento, emissão de pareceres, promoções ou participação em comitês consultivos ou diretivos, entre outras, que pudessem influenciar no desenvolvimento do trabalho.

\section{Referências}

1 - IOANNIDES-DEMOS, L. L.; PICCENNA L.; MCNEIL J. J. Pharmacotherapies for Obesity: Past, Current, and Future Therapies. Journal of Obesity, v.17, p. 96-74, 2011.

Disponível

em:

<https://www.hindawi.com/journals/jobe/2011/179674/>

. Acesso em: 08 mai, 2017.

2 - BOTSARIS, A. S. Fitoterapia chinesa e plantas brasileiras. São Paulo: Editora Ícone, 1995, p.550.

3 - SOUZA, A. D. Z.; MEDIETA, M. C.; CEOLIN, T.; HECK, R. M. As plantas medicinais como possibilidade de cuidado para distúrbios urinários. Revista de Enfermagem da UFSM, v. 4, n. 2, p. 342-349, 2014. Disponível em:

https://periodicos.ufsm.br/reufsm/article/view/10377>. Acesso em: 22 jun, 2018.

4 - LIMA, F. A.; BÚ, E. A. D.; SOARES, M. P.; ARAUJO, C. R. F. A fitoterapia e sua inserção no contexto da atenção básica. Revista Saúde \& Ciência Online, v. 4, n. 2, p. 120-128, 2015. Disponível em: < http://www.ufcg.edu.br/revistasaudeeciencia/index.php/

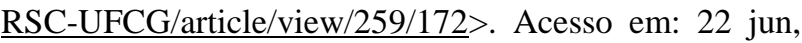
2018.

5 - BRUNING, M. C. R.; MOSEGUI, G.B.G.; VIANNA, C. M. M. A utilização da fitoterapia e de plantas medicinais em unidades básicas de saúde nos municípios de Cascavel e Foz do Iguaçu - Paraná: a visão dos profissionais de saúde. Ciência \& Saúde Coletiva, v.17, n.10, p.2675-2685, 2012. Disponível em: < http://www.cienciaesaudecoletiva.com.br/artigos/autilizacao-da-fitoterapia-e-de-plantas-medicinais-emunidades-basicas-de-saude-nos-municipios-de-cascavele-foz-do-iguacu-parana-a-visao-dos-profissionais-desaude/9266?id=9266>. Acesso em: 22 jun, 2018.

6 - PIRIZ, M. A.; LIMA, C. A. B.; JARDIM, V. M. R.; MESQUITA, M. K.; SOUZA, A. D. Z.; HECK, R. M. Plantas medicinais no processo de cicatrização de feridas: uma revisão de literatura. Revista Brasileira de Plantas Medicinais, v.16, n.3, p.628-636, 2014. Disponível em: <http://www.scielo.br/scielo.php?pid=S1516- 
05722014000300020\&script=sci_abstract\&tlng=pt $>$.

Acesso em: 22 jun, 2018.

7 - ARAUJO, W. R. M.; SILVA, R. V.; BARROS, C. S.;

AMARAL, F.M.M. Inserção da fitoterapia em unidades de saúde da família de São Luís, Maranhão: realidade, desafios e estratégias. Revista Brasileira de Medicina de Família e Comunidade, v. 9, n. 32, p. 258-263, 2014. Disponível em:

https://www.rbmfc.org.br/rbmfc/article/view/789>.

Acesso em: 22 jun, 2018.

8 - SAMPAIO, L. A.; OLIVEIRA, D. R.; KERNTOPF, M. R.; JUNIOR, F.E. B.; MENEZES, I. R. A. Percepção dos enfermeiros da estratégia saúde da família sobre o uso da fitoterapia. Revista Mineira de Enfermagem, v. 17, n. 1, p. 77-85, 2013. Disponível em: < http://www.reme.org.br/artigo/detalhes/580>. Acesso em: 22 jun, 2018.

9 - MACHADO, D. C.; CZERMAINSKI, S. B.

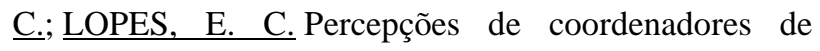
unidades de saúde sobre a fitoterapia e outras práticas integrativas e complementares. Saúde em Debate, vol.36, n.95, p.615-623, 2012. Disponível em: < http://www.scielo.br/scielo.php?pid=S0103$\underline{11042012000400013 \& \text { script }=\text { sci abstract\&tlng }=\mathrm{pt}}>$. Acesso em: 22 jun, 2018.

10 - CEOlin, T.; CEOLIN, S.; BONOW, C. T.; VARGAS, N. S. C.; MINUTO, J.C.; LOPES, C.V. Relato de experiências do curso de plantas medicinais para profissionais de saúde. Revista Baiana de Saúde Pública, v.32, n.2, p. 501, 2013. Disponível em: < http://ojs.unesp.br/index.php/revista_proex/article/view/1 565>. Acesso em: 22 jun, 2018.

11 - DISTASI, L.C. Plantas medicinais: verdades e mentiras, o que os usuários e os profissionais de saúde precisam saber. 1.ed. São Paulo: UNESP, p. 133, 2007.

12 - ANTONiO, G. D.; TESSER, C. D.; MORETTIPIRES, R. O. Fitoterapia na atenção primária à saúde. Revista de Saúde Pública, v. 48, n. 3, p. 541-553, 2014. Disponível em: < http://www.scielo.br/scielo.php?pid=S0034$\underline{89102014000300541 \& \text { script }=\text { sci abstract\&tlng }=p t>.}$ Acesso em: 22 jun, 2018.
13 - ARAUJO, M. A. R. Unidades de Conservação no Brasil: da república à gestão de classe mundial. Belo Horizonte: SEGRAC, 2007.

14 - BARREIRO, E. J.; BOLZANI, V. S. Biodiversidade: fonte potencial para a descoberta de fármacos. Química Nova, São Paulo, v. 32, no. 3, p. 679-88, 2009. Disponível em:

http://quimicanova.sbq.org.br/detalhe artigo.asp?id=333 >. Acesso em: 22 jun, 2018.

15 - BRASIL. Ministério da Saúde. Conselho Nacional de Saúde. Resolução no 466/12. Dispõe sobre pesquisas envolvendo seres humanos. Disponível em: <http://bvsms.saude.gov.br/bvs/saudelegis/cns/2013/res0 466_12_12_2012.html>, Acesso em: 16 mai, 2018.

16 - DAMASCENO, E. M. A. Fitoterapia e Profissionais da Saúde na Estratégia de Saúde Da Família. Dissertação Mestrado, Universidade Estadual de Montes Claros, Montes Claros, 2016.

17 - FEITOSA, M. H. A.; SOARES, L. L.; BORGES, G. A.; ANDRADE, M. M.; COSTA, S. M. Inserção do Conteúdo Fitoterapia em Cursos da Área de Saúde. Revista Brasileira de Educação Médica, v. 40, n. 2, p. 197-203, 2016. Disponível em: < http://www.scielo.br/scielo.php?pid=S0100$\underline{55022016000200197 \& \text { script }=\text { sci abstract\&tlng }=\mathrm{pt}}>$.

Acesso em: 14 jun, 2018.

18 - MOURA, A. S. C.; ARAÚJO, L. G.; BRANCO, A. C. S. C.; CARVALHO, L. M. F. Conhecimento sobre plantas medicinais e fitoterápicos: um estudo com acadêmicos de nutrição. Revista Interdisciplinar, v. 9, n. 3, p. 18-25, 2016. Disponível em: < https://revistainterdisciplinar.uninovafapi.edu.br/index.p hp/revinter/article/view/1027>. Acesso em: 22 jun, 2018. 19 - MAYA, M. V. P. O conhecimento dos acadêmicos de enfermagem a respeito do uso de fitoterápicos. Monografia, Centro de Ensino Unificado de Brasília, Brasília. 2016. Disponível em: < http://repositorio.uniceub.br/handle/235/11052>. Acesso em: 22 jun, 2018.

20 - JÚNIOR, B. J. N.; TÍNEL, L. O.; SILVA, E. S.; RODRIGUES, L. A.; FREITAS, T. O. N.; NUNES, X. P.; AMORIM, E. L. C. Avaliação do conhecimento e 26 | P á g i n a 
A utilização da fitoterapia e plantas medicinais como prática terapêutica pela visão de estudantes de enfermagem

percepção dos profissionais da estratégia de saúde da família sobre o uso de plantas medicinais e fitoterapia em Petrolina-PE, Brasil. Revista Brasileira de Plantas Medicinais, Campinas, v. 18, n. 1, p. 57-66, 2016. Disponível em: http://www.scielo.br/pdf/rbpm/v18n1/1516-0572-rbpm18-1-0057.pdf>. Acesso em: 22 jun, 2018.

21 - FERREIRA, E. T.; DOS SANTOS, E. S.; MONTEIRO, J. S.; GOMES, M. D. S. M.; DE OLIVEIRA MENEZES, R. A.; DE SOUZA, M. J. C. A utilização de plantas medicinais e fitoterápicos: uma revisão integrativa sobre a atuação do enfermeiro. Brazilian Journal of Health Review, v.2, n.3, p.1511-1523, 2019. Disponível em: <http://www.brjd.com.br/index.php/BJHR/article/view/1 383> Acesso em: 29 abr, 2019.

22 - FARIA, A. M. B.; VALIATTI, T. B.; OLIVEIRA, A. A.; SALVI, J. O. A fitoterapia entre acadêmicos das ciências da vida. Revista Saúde e Desenvolvimento, v.11, n.9, p.198-213, 2017. Disponível em: <https://www.uninter.com/revistasaude/index.php/saude Desenvolvimento/article/view/786/469>. Acesso em: 22 jun, 2018.

23 - ZENI, A. L. B.; PARISOTTO, A. V.; MATTOS, G.; SANTA HELENA, E. T. Utilização de plantas medicinais como remédio caseiro na Atenção Primária em Blumenau, Santa Catarina, Brasil. Ciência \& Saúde Coletiva, v. 22, n. 8 , p. 2703-2712, 2017. Disponível em: < http://www.scielo.br/pdf/csc/v22n8/1413-8123-csc-2208-2703.pdf>. Acesso em: 05 jun, 2018.

24 - ÂNGELO, T.; RIBEIRO, C. C. Utilização de plantas medicinais e medicamentos fitoterápicos por idosos.

Revista Ciência e Desenvolvimento, Vitória da Conquista, v.7, n.1, p.18-31, 2014. Disponível em: < http://srv02.fainor.com.br/revista/index.php/memorias/ar ticle/view/246/188>. Acesso em: 12 jun, 2018.

25 - COSTA, R. S.; BRASIL, T. C.; SANTOS, C. J.; SANTOS, D. B.; BARRETO, M. L.; NEVES, N. M. A.; FIGUEIREDO, C. A. V. Produtos naturais utilizados para tratamento de asma em crianças residentes na cidade de Salvador-BA, Brasil. Revista Brasileira de Farmacognosia, Curitiba, v. 20, n. 4, p. 594-599, 2010. Disponível em: http://www.scielo.br/scielo.php?script=sci_arttext\&pid= S0102-695X2010000400020>. Acesso em: 22 jun, 2018. 26 - LOPES, G. F. G.; PANTOJA, S. C. S. Levantamento das espécies de plantas medicinais utilizadas pela população de Santa Cruz - Rio de Janeiro - RJ. Revista Eletrônica Novo Enfoque, v. 15, edição especial, p. 76 82, 2012. Disponível em: <http://www.castelobranco.br/sistema/novoenfoque/files/ 15/artigos/15_Levantamentodasespeciesdeplantas_Sonia Pantoja_Subtitulo_VF.pdf>. Acesso em: 04 jun, 2018.

27 - DE ARAÚJO, M. S. C.; DA COSTA, J. W.; DA COSTA, A. A.; DE LIMA TOCCHIO, P. S. P.; DE AZEVEDO ARAÚJO, L. S.; DE ARAÚJO NUNES, V. M. A utilização de plantas medicinais e da fitoterapia em comunidades assistidas pela Estratégia Saúde da Família. Revista Brasileira de Pesquisa em Saúde/Brazilian Journal of Health Research, v.17, n.4, p.6-16, 2016. Disponível em: <http://periodicos.ufes.br/RBPS/article/view/14325> Acesso em: 29 abr, 2019.

28 - SZERWIESKI, L. L. D.; CORTEZ, D.; BENNEMANN, R. M.; SILVA, E. S.; CORTEZ L. E. R. Uso de plantas medicinais por idosos da atenção primária.

Revista Eletrônica de Enfermagem, v.19, 2017. Disponível em: <http://dx.doi.org/10.5216/ree.v19.42009>. Acesso em: 25 jan, 2018.

29 - JACOBI, C. S.; BRACCINI, V.; SILVA, S. T. A.; CERETA, L. R.; CASTILHOS, L.; PIRES, M. G.; PASINI, L. C.; SORDI, R.; ROESE, A.; CEOLIN, T. Relato de experiência sobre o uso de plantas medicinais por uma comunidade do município de Uruguaiana/RS. Journal of Nursing and Health, v.1, n.2, p.334-345, 2011. Disponível em: <https://periodicos.ufpel.edu.br/ojs2/index.php/enfermag em/article/view/3510>. Acesso em: 22 jun, 2018.

30 - NUNES, J. D.; MACIEL, M. V. A importância da informação do profissional de enfermagem sobre o cuidado no uso das plantas medicinais: uma revisão de literatura. Revista Fitos Eletrônica, v. 10, n. 4, p. 518525, 2017. Disponível em: < http://webcache.googleusercontent.com/search?q=cache: http://revistafitos.far.fiocruz.br/index.php/revistafitos/article/view/385>. Acesso em: 10 jan, 2018. 
A utilização da fitoterapia e plantas medicinais como prática terapêutica pela visão de estudantes de enfermagem

31 - McCABE, P. Complementary and alternative medicine in Australia: a contemporary overview. Complementary Therapies in Clinical Practice, v.11, n.1, p.28e31, 2005. Disponível em: <https://www.sciencedirect.com/science/article/pii/S174 4388104000866?via\%3Dihub> Acesso em: $30 \mathrm{abr}, 2019$. 32 - BOOTH-LAFORCE, C.; SCOTT, C.S.; HEITKEMPER, M.M.; CORNMAN, B.J.; LANA, M.C.; BOND, E.F.; SWANSON, K.M. Complementary and alternative medicine (CAM) attitudes and competencies of nursing students and faculty: results of integrating CAM into the nursing curriculum. Journal of professional Nursing, v.26, n.5, p.293e300, 2010. Disponível em:

https://www.ncbi.nlm.nih.gov/pmc/articles/PMC294734

9/pdf/nihms-190998.pdf> Acesso em: 30 abr. 2019.

33 - BALOUCHI A.; MAHMOUDIRAD, G.; HASTINGS-TOLSMA, M.; SHOROFi, S.A.; SHAHDADI, H.; ABDOLLAHIMOHAMMAD, A. Knowledge, attitude and use of complementary and alternative medicine among nurses: A systematic review.

Complementary Therapies in Clinical Practice, v.31, p.146e157, 2018. Disponível em: < https://www.sciencedirect.com/science/article/pii/S1744 388118300483?via\%3Dihub> Acesso em 30 abr. 2019. 34 - FONTENELE, R. P.; CARVALHO, A. L. M.; OLIVEIRA, F. A. Fitoterapia na Atenção Básica: olhares dos gestores e profissionais da Estratégia Saúde da Família de Teresina (PI), Brasil. Ciência \& Saúde Coletiva, v.18, n.8, p.2385-2394, 2013. Disponível em: <http://www.scielo.br/scielo.php?pid=S1413$\underline{81232013000800023 \& \text { script }=\text { sci abstract }>}$. Acesso em: 10 jan, 2018.

35 - SILVA, L. B.; LIMA, I. C.; BASTOS, R. A. Terapias complementares e integrativas: conhecimento e utilização pelos docentes do curso de enfermagem de uma instituição pública. Revista de Saúde Coletiva da UEFS, v. 5, n. 1, p. 40-45, 2016. Disponível em: < http://periodicos.uefs.br/index.php/saudecoletiva/article/ view/1008>. Acesso em: 29 mai, 2018. 